\title{
Fluid Flow and Mixing Process in a Bottom Stirring Electrical Arc Furnace with Multi-plug
}

\author{
Baokuan LI \\ Department of Thermal Engineering, The School of Materials and Metallurgy, Northeastern University, Shenyang, 110006 \\ China, visiting researcher, Department of Metallurgy, Graduate School of Engineering, The University of Tokyo, Hongo, \\ Bunkyo-ku, Tokyo 113-8656 Japan.
}

(Received on December 22, 1999; accepted in final form on May 23, 2000)

\begin{abstract}
Fluid flow and mixing process in a bottom stirring electrical arc furnace (EAF) with the single- and multiplug are experimentally and numerically studied. A homogeneous fluid with a spatially variable density is used to model the gas-liquid flow. $k-\varepsilon$ turbulence model is employed to calculate the effective viscosity. The five layouts are selected to examine the mixing times. The mixing time is defined as the function of the diameter, number, location of plugs and the tracer injection point. The results show that the variation of mixing time is not obvious with the increasing of plug diameter, but the stirring efficiency is getting better when plug number is increased and distributed in deep and off-central region in vessel. This is because the angular velocities occur and the more stirring energy is obtained in such layouts.
\end{abstract}

KEY WORDS: bottom stirring electrical arc furnace (EAF); fluid flow and mixing process; mathematical model.

\section{Introduction}

The electrical arc furnaces are mainly used to produce the special steel. The electrical Arc is used for the heat source, the electromagnetic force, which is produced by effect of the electromagnetic field on molten steel, is employed to stir melt in baths. Nevertheless, the stirring energy can not meet the need of practical high efficiency operation. In order to reinforce heat and mass transfer, promote the reactive rate, and remove effectively the non-metallic inclusions, the bottom stirring technique of ladle operation is transplanted to the process of EAF operation. The key of operation is to obtain high stirring efficiency by selecting the optimum injection mode. Author and many researchers ${ }^{1-4)}$ have reported previous works on the bottom stirring ladles. In particular, Castillejos and Brimacombe ${ }^{5)}$ found that the radical gas fraction profiles, at different axial positions in the plume, exhibit similarity. Joo and Guthrie ${ }^{6}$ had studied the flows and mixing in steelmaking ladles designed for single- and dual-plug bubbling operation. However, the EAF operation has the following features by contrast with ladles.

(1) The raw material loaded in furnace is solid and porous, so the liquid bath after melting is shallow;

(2) To reduce the effect of pouring caused by upwelling plume on electrical arc stability, the multi-plug is used to inject the gas;

(3) The slope angle of the sidewall of EAF is large.

In order to improve the stirring efficiency, it is necessary to understand the flow characteristics and mixing mechanism in the bottom stirring EAF.
The purpose of the present research is to explore the optimum layouts of plug design and furnace operation. A mathematical model has been developed to simulate the fluid flow and mixing in the bottom stirring process of EAF, and a water model experiment has been carried out to measure the velocities and mixing times for different layouts about distribution of plugs in the bottom stirring EAF.

\section{Experiment}

\subsection{Experimental Procedures}

A schematic diagram of the equipment used to measure local mixing times is shown in Fig. 1. Experiment has been carried out in a one seventh water model of a typical 30-ton EAF. The model has a bottom diameter of $0.3 \mathrm{~m}$, a top diameter of $0.5 \mathrm{~m}$, and a height of $0.1 \mathrm{~m}$ water, this corresponds to the actual (internal) dimensions of $2.1 \mathrm{~m}, 3.5 \mathrm{~m}$, and $0.7 \mathrm{~m}$, respectively. The vessel itself is housed in a much larger rectangular tank filled with water to the same level as $0.1 \mathrm{~m}$ height and fitted with transparent sidewall. Visual distortion of plumes caused by the curved sidewall of the EAF could thereby be eliminated in case that a laser Doppler velocimeter is used to measure the velocities.

There are seven porous plugs in the bottom of the EAF, one of them is located at center and its diameter is changeable by interlocking screw, three of them are uniformly distributed $(\pi / 3, \pi, 5 \pi / 3)$ at circle of electrode $(0.4 R)$, the others are uniformly $(\pi / 3, \pi, 5 \pi / 3)$ distributed at inclined wall. Before each experiment, air hoses would be connected to the desired plug(s). Tracer is $0.216 \mathrm{~mol} \mathrm{KCl}$ solution, the injection points are at $(0.65 R, 0,0.2 H),(0.65 R, \pi / 6,0.2 H)$, 


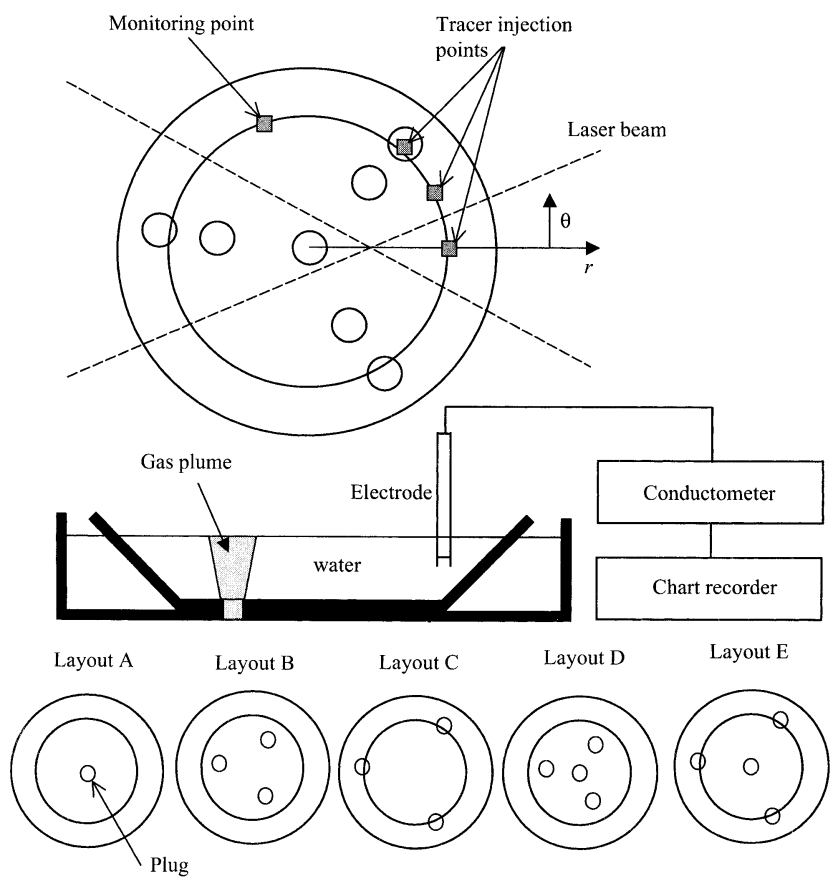

Fig. 1. Schematic of experimental system for measurements of the mixing times and fluid velocities.

and $(0.65 R, \pi / 3,0.2 H)$, respectively. The diameter of plugs is $3 \times 10^{-3} \mathrm{~m}$, but a plug at center is changeable from $3 \times$ $10^{-3} \mathrm{~m}$ to $10 \times 10^{-3} \mathrm{~m}$. The monitoring point is at $(0.65 R$, $2 \pi / 3,0.2 H$ ), which is the relatively quiescent point. The $95 \%$ bulk mixing time criterion is then selected as a suitable standard mixing time, this is defined as that time when all the local concentrations of tracer addition have reached $95 \%$ of the bulk well-mixed value.

Mixing times are measured five to six times for a given set of experimental conditions, the averaged value then being reported as the measured mixing time. It is appropriate to note that individual mixing times fall within $10 \%$ of the averaged values reported here.

\subsection{Experimental Results}

Figure 2 illustrates that the measured mixing times vary with tracer injection point for layout $\mathrm{B}$ when the airflow rate is $3 \mathrm{~N} l / \mathrm{min}$. The long mixing time can be seen when tracer injection point is located at center of the bow between two plugs. This is because the point is far from plugs and local fluid velocities are relatively small. Thus this location is chosen as constant tracer injection point for other conditions.

Figure 3 shows the effect of airflow rate on mixing times for various layouts. The mixing times for layout B and D are short, and that of layout $\mathrm{C}$ is the longest.

The measured two-dimensional velocities in selected ver-

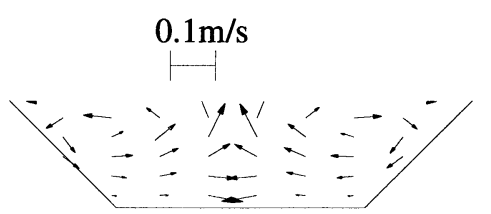

(a)

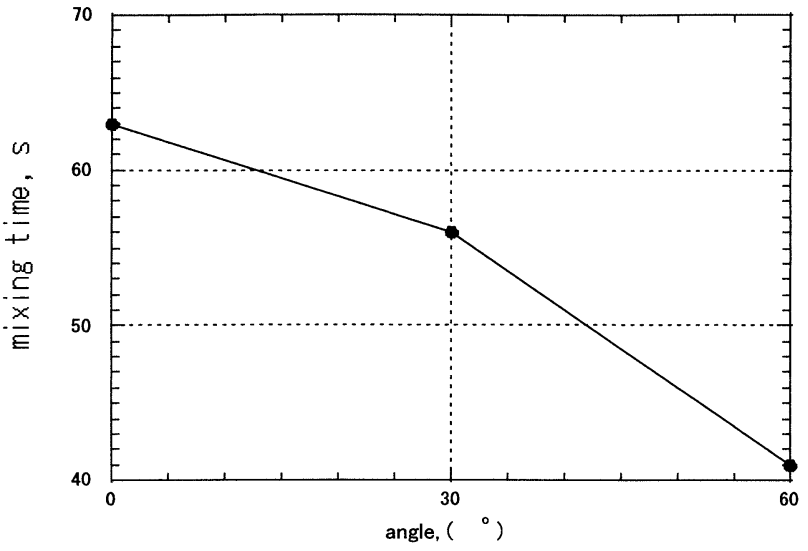

Fig. 2. Effect of the angle of tracer injection point on the mixing time for layout $\mathrm{B}, Q=3 \mathrm{Nl} / \mathrm{min}$. $(r=0.65 R, Z=0.5 \mathrm{H}$, and plug diameter is $3 \times 10^{-3} \mathrm{~m}$ )

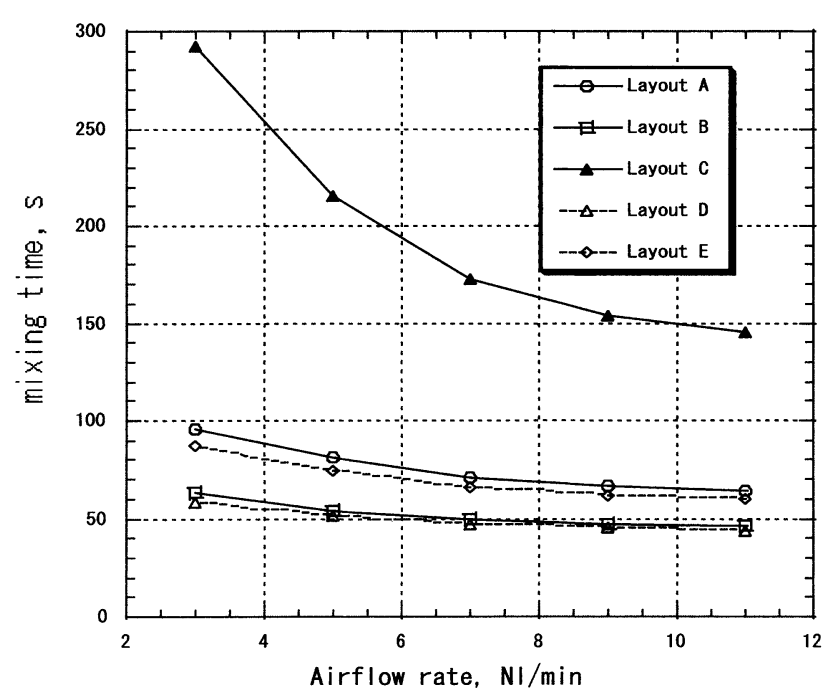

Fig. 3. Effect of the airflow rate on the mixing time for various layouts, diameter of plugs is $3 \times 10^{-3} \mathrm{~m}$.

tical sections for layout A and B are shown in Fig. 4. By ordering the measured results, it can be found that the velocities tend to be uniform in space in the case of multi-plug injection.

\section{Mathematical Formulation}

\subsection{Governing Equations}

In order to describe fluid flow, turbulent properties, and tracer dispersion in the bottom stirring EAF during oxidizing and reducing period, the following assumptions are made in the mathematical model:

(1) The electromagnetic force is neglected. The power supplied by alternating current for EAF is stopped com-

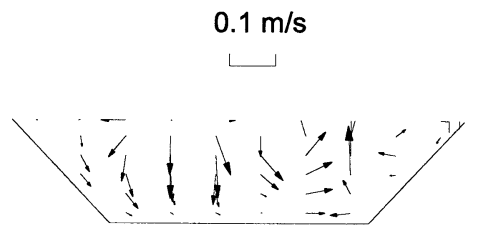

(b)

Fig. 4. Measured flow velocities on main vertical section in water model, (a) layout A and (b) layout B. 
Table 1. Variables in the common equation.

\begin{tabular}{|c|c|c|c|}
\hline & $\phi$ & $\Gamma$ & $S_{\phi}$ \\
\hline Continuity & 1 & 0 & 0 \\
\hline Axial momentum & $u$ & $\mu_{\mathrm{e}}$ & $S_{u}=-\frac{\partial p}{\partial z}-\rho g+\frac{\partial}{\partial z}\left(\mu_{\mathrm{e}} \frac{\partial u}{\partial z}\right)+\frac{1}{r} \frac{\partial}{\partial r}\left(r \mu_{\mathrm{e}} \frac{\partial v}{\partial z}\right)+\frac{1}{r} \frac{\partial}{\partial \theta}\left(\mu_{\mathrm{e}} \frac{\partial w}{\partial z}\right)$ \\
\hline Radial momentum & $\mathrm{V}$ & $\mu_{\mathrm{e}}$ & $\begin{aligned} S_{v}= & -\frac{\partial p}{\partial r}+\frac{\partial}{\partial z}\left(\mu_{\mathrm{e}} \frac{\partial u}{\partial r}\right)+\frac{1}{r} \frac{\partial}{\partial r}\left(r \mu_{\mathrm{e}} \frac{\partial v}{\partial r}\right)+\frac{1}{r} \frac{\partial}{\partial \theta}\left[\mu_{\mathrm{e}} \frac{\partial}{\partial r}\left(\frac{w}{r}\right)\right] \\
& +\left[\frac{\rho w^{2}}{r}-\frac{2 \mu_{\mathrm{e}}}{r^{2}}\left(v+\frac{\partial w}{\partial \theta}\right)\right]\end{aligned}$ \\
\hline Angular momentum & $W$ & $\mu_{\mathrm{e}}$ & $\begin{aligned} S_{w}= & \left.-\frac{1}{r} \frac{\partial p}{\partial \theta}+\frac{\partial}{\partial z}\left(\mu_{\mathrm{e}} \frac{\partial u}{r \partial \theta}\right)+\frac{1}{r} \frac{\partial}{\partial r}\left[\mu_{\mathrm{e}} \frac{\partial v}{\partial \theta}-w\right)\right] \\
& +\frac{1}{r} \frac{\partial}{\partial \theta}\left[\frac{\mu_{\mathrm{e}}}{r}\left(\frac{\partial w}{\partial \theta}+2 v\right)\right]+\left[\frac{\mu_{\mathrm{e}}}{\mathrm{r}}\left(\frac{\partial w}{\partial r}+\frac{1}{r} \frac{\partial v}{\partial \theta}-\frac{w}{r}\right)-\frac{\rho v w}{r}\right]\end{aligned}$ \\
\hline $\begin{array}{l}\text { Turbulent kinetic } \\
\text { Energy }\end{array}$ & $k$ & $\frac{\mu_{t}}{\sigma_{k}}$ & $\begin{aligned} S_{k}= & G-\rho \varepsilon \\
G & =2 \mu_{l}\left[\left(\frac{\partial u}{\partial z}\right)^{2}+\left(\frac{\partial v}{\partial r}\right)^{2}+\left(\frac{\partial w}{r \partial \theta}+\frac{v}{r}\right)^{2}\right]+\mu_{t}\left[\left(\frac{\partial u}{\partial r}+\frac{\partial v}{\partial z}\right)^{2}\right. \\
& \left.+\left(\frac{\partial w}{\partial z}+\frac{\partial u}{r \partial r}\right)^{2}+\left(\frac{\partial v}{r \partial \theta}+\frac{\partial w}{\partial r}-\frac{w}{r}\right)^{2}\right]\end{aligned}$ \\
\hline $\begin{array}{l}\text { Dissipation rate of } \\
\text { turbulent kinetic energy }\end{array}$ & $\varepsilon$ & $\frac{\mu_{t}}{\sigma_{\varepsilon}}$ & $S_{\varepsilon}=C_{1} G \frac{\varepsilon}{k}-C_{2} \rho \frac{\varepsilon^{2}}{k}$ \\
\hline Dispersion of tracer & $C$ & $\Gamma_{t, C}$ & $S_{C}=-\frac{\partial}{\partial t}(\rho C)$ \\
\hline \multicolumn{4}{|c|}{$\mu_{t}=\frac{C_{\mu} \rho k^{2}}{\varepsilon}, \mu_{\mathrm{e}}=\mu+\mu_{t}, \quad \Gamma_{t, C}=\frac{\mu}{\sigma}+\frac{\mu_{t}}{\sigma_{t, c}}$} \\
\hline \multicolumn{4}{|c|}{$C_{1}=1.44, C_{2}=1.92, C_{\mu}=0.09, \sigma_{k}=1.0, \sigma_{\varepsilon}=1.3, \sigma_{t, C}=1.0$} \\
\hline
\end{tabular}

monly during gas injection, so that this assumption can be accepted.

(2) The whole domain is treated as a homogeneous medium, but with a spatially variable density. The interact force between air and liquid is not considered.

(3) Flow is induced by the density differences between the gas/liquid plume region and pure liquid region

(4) Surface waves and attendant oscillations are neglected. For one plug, the height of plume may be high, but it will tend to plat when the multi-plug is employed.

The relevant partial differential equations requiring solution are the equations of continuity, momentum, and mass conservation, expressed in a three-dimensional cylindrical, incompressible, and ensemble-averaged form. These can be written in the following common form:

$$
\begin{aligned}
& \frac{\partial}{\partial z}(\rho u)+\frac{1}{r} \frac{\partial}{\partial r}(r \rho v \phi)+\frac{1}{r} \frac{\partial}{\partial \theta}(\rho w \phi) \\
& \quad=\frac{\partial}{\partial z}\left(\Gamma \frac{\partial \phi}{\partial z}\right)+\frac{1}{r} \frac{\partial}{\partial r}\left(r \Gamma \frac{\partial \phi}{\partial r}\right)+\frac{1}{r} \frac{\partial}{\partial \theta}\left(\Gamma \frac{1}{r} \frac{\partial \phi}{\partial \theta}\right)+S_{\phi}
\end{aligned}
$$

The equations for each variable can be get in Table 1 .

The density of the plume can be obtained by

$$
\rho=\rho_{\mathrm{g}} \alpha+\rho_{\mathrm{L}}(1-\alpha)
$$

where gas volume fraction $\alpha$ can be calculated by the experiment of Castilljeos and Brimacombe ${ }^{5)}$

$$
\alpha=\alpha_{\max } \exp \left(-0.7\left(\frac{r}{r_{\alpha_{\max 2}}}\right)^{2.4}\right)
$$

$\alpha_{\max }$ is the maximum value of the local gas volume fraction. $r_{\alpha_{\max 2}}$, termed the half-value radius, is distance from the axis of symmetry to the position where the gas fraction is half the maximum value. The gas-liquid plume has the form of a regular cone with its origin some distance upstream of the plug mouth. The angle subtended by the cone varied between 18 degree and 22 degree. Generally, the bath of the EAF is shallow, the interaction of plumes should be avoided so as to prevent the decrease of stirring efficiency in the arrangement of plugs.

Although the gas-liquid plume is included in the flow field, The standard $k-\varepsilon$ model of turbulence proposed by Jones and Launder ${ }^{7)}$ was used still to determine the turbulent viscosity due to lack the model of the two phase fluid turbulence. The reason is that the effect of plume on the effective viscosity has partly been taken into account by the variable density, and gas volume fraction is very little in the bulk fluid.

As the dispersion equation of tracer is linear, its non-dimensional equation is same as the original one. The initial value of concentration at tracer injection point is set to $100 \%$, other region is set to zero.

The mixing time is the time when monitoring point concentration is coincidence with the following equation:

$$
\left|\frac{C-C_{\mathrm{f}}}{C_{\mathrm{f}}}\right| \leq 0.05
$$

where $C_{\mathrm{f}}$ is the final uniformity concentration.

\subsection{Boundary Conditions}

Impermeable conditions are assumed at solid walls, i.e. all variables (i.e. $u, v, w, k$, and $\varepsilon$ ) at solid walls are set to zero. At the node adjacent to solid boundaries, the wall shear stress and the energy dissipation rate are calculated by using the wall function method. ${ }^{7)}$ At the free surface, normal velocity components and normal gradients of all variables are set equal to zero. 
Table 2. Effects of diameter on mixing times for single plug $(Q=3 \mathrm{~N} l / \mathrm{min})$.

\begin{tabular}{ccccc}
\hline Plug diameter, $\mathrm{m}$ & $3 \times 10^{-3}$ & $6 \times 10^{-3}$ & $10 \times 10^{-3}$ & $30 \times 10^{-3}$ \\
\hline Measured mixing time, s & 96 & 94 & 91 & - \\
Computed mixing time, s & 102 & 97 & 95 & 81 \\
\hline
\end{tabular}

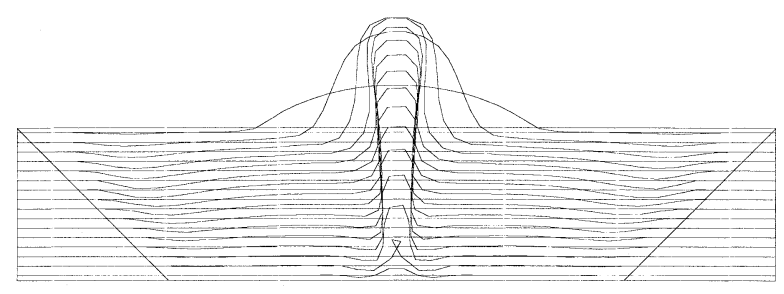

(a)

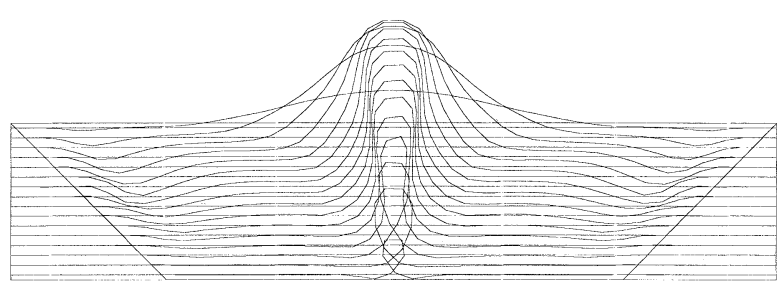

(b)

Fig. 5. Computed vertical velocity top vector curves, (a) $d=$ $0.003 \mathrm{~m}$ and (b) $d=0.03 \mathrm{~m}$.

\subsection{Solution Procedures}

As the mixing process of tracer depends on the flow field in the furnace, the fluid flow and turbulence equations are first solved, and then the results are based to solve the tracer dispersion equation. The discretized equations were derived from the integral form of the original conservation equations, a power-law difference scheme was used to approximate the convection and diffusion terms. the staggered grid system was used to store the velocity components so as to suppress the pressure fluctuation. The size of main grid is $18 \times 54 \times 18$ in $r, \theta$, and $z$ direction, respectively. The stairway-shaped approximation was adopted to the treatment of the inclined wall. The SIMPLEC Algorithm solves the group of equations, the ADI solver associated with the block correction technique are used to solve the algebraic equations. The convergence criterion of flow field is designated as the mass source of continuity equation reduced to $0.01 \%$ of initial value.

\section{Numerical Simulation}

In order to study stirring efficiency during such gas injection operation in the EAF, computations are performed on water model so as to analyze the mixing behavior for all layouts. First of all, the influence of a plug diameter on mixing process is examined in Table 2. An air flow rate of $3 \mathrm{Nl} / \mathrm{min}\left(5.0 \times 10^{-5} \mathrm{~m}^{3} / \mathrm{s}\right)$ is used in these cases. For single plug, when the plug diameter is twice, or triple as much as the original one, whether experimental or computational, the variation of mixing times is not obvious. The remarked reducing of mixing time occurs in case that a plug diameter is ten times as much as the original one. Figure 5 represents the vector top curves for computed vertical velocity for small and large plug diameter. As the expected, the up-

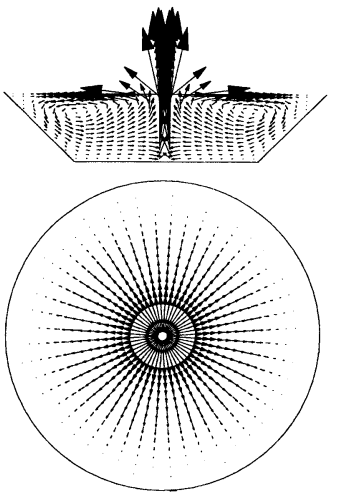

(a)
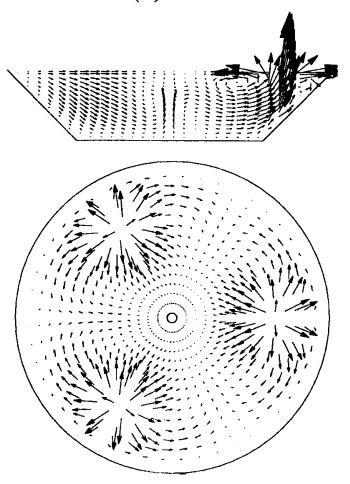

(c)
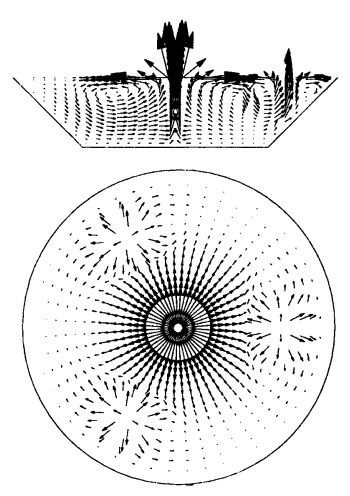

(e)
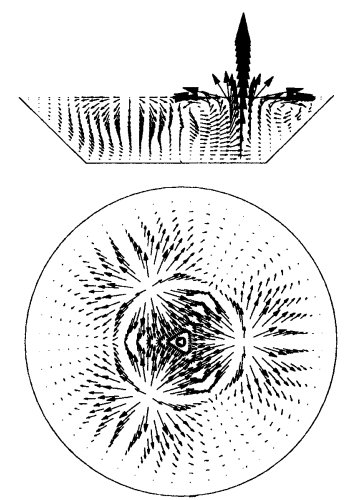

(b)
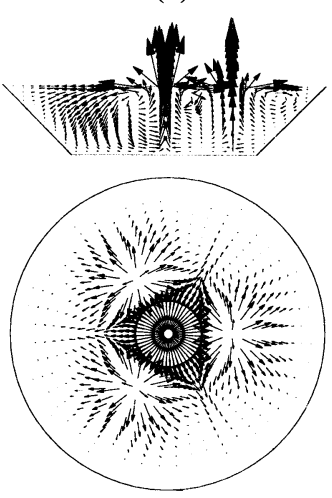

(d)
Fig. 6. Computed velocity vectors in main vetical section and free surface of the EAF, $Q=3 \mathrm{~N} l / \mathrm{min}, d=3 \times 10^{-3} \mathrm{~m}$. (a) Layout A, (b) layout B, (c) layout C, (d) layout D, and (e) layout E.

welling plume zone is obviously widened in the case of large plug diameter $(0.03 \mathrm{~m})$. It is difficult to solve the pouring of water into the gas piping in laboratory, so that the test for the large diameter $(0.03 \mathrm{~m})$ is not carried out.

It is efficient to improve stirring condition in vessel when the multi-plug is employed to inject gas. Computations are performed on five layouts in Fig. 1. Figure 6 illustrates some predicted two-dimensional flow fields in main vertical section $(\theta=0)$ and free surface for all layouts. Figure 7 shows the comparison of vertical velocities on vertical section of water model for layout A between computed and measured, which are located at (a) near the free surface and (b) half height. It can be found there is a good agreement between them. The flow field of the EAF with multi-plug, which is distributed in deep and off-central region of bottom stirring EAF, is favorable for the mixing of tracer. For a 


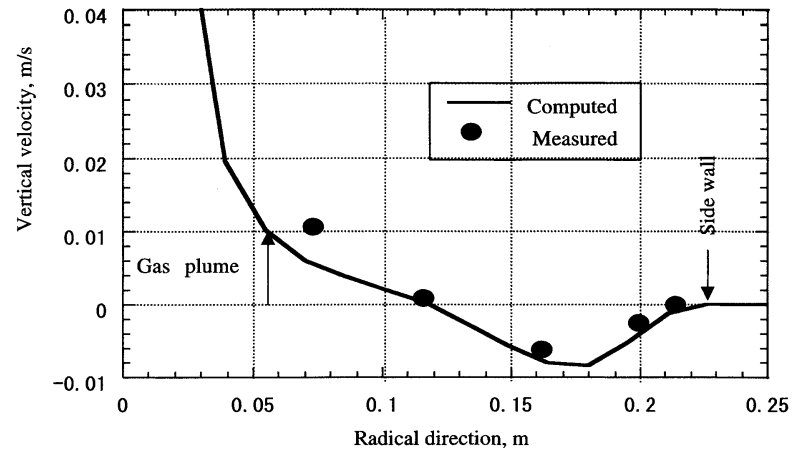

(a)

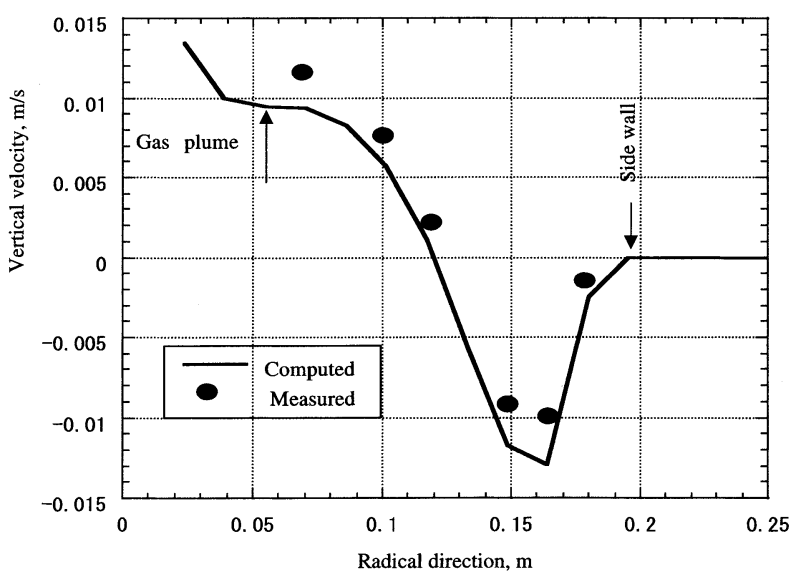

(b)

Fig. 7. Vertical velocities on vertical section of a EAF water model (layout A, $Q=3 \mathrm{~N} l / \mathrm{min}$ ), (a) $Z=0.9 \mathrm{H}$, and (b) $Z=$ $0.5 \mathrm{H}$. single central plug, the angular velocity is zero, the angular mixing depends only on the molecular and turbulent diffusion. However, the angular velocities occur in flow fields for the off-centered injection as shown in Fig. 6 (b, c, d, and e), which significantly affect tracer dispersion rates. Figures 8 and 9 exhibit tracer concentration fields at different time for layout $\mathrm{A}$ and $\mathrm{B}$, respectively. The mixing processes accord with the characteristics of flow fields. By contrast the computed mixing times with the measured for five layouts in Table 3, a good agreement is obtained. The predicted mean velocities within the vessel vary with plug number and location, which are presented in Table 4. It can be seen that the mean velocities in angular, axial, and radial directions become close to each other for multi-plug distributed in deep and off-central region. The total mean velocities or the total stirring energy for layout A is the highest, however, as the angular velocity is zero, the mixing efficiency is low. Although the total momentum of the layouts $\mathrm{B}$ or $\mathrm{D}$ is lower than that of the layout $\mathrm{A}$, which is decided by the low total mean velocities, but the mean velocity components in all direction are equal approximately to one another. Therefore, the stirring efficiency is relatively high. If the other factors such as surface stability are considered in the furnace design, the layout D is superior to B. But the number of plugs in layout $\mathrm{D}$ is more than in $\mathrm{B}$, so that the selection in design must be made in the light of practical requirement. As for layout $\mathrm{C}$, the enough stirring energy can not be obtained by fluid, since the plugs are located in the inclined wall with a short floatation path of gas bubbles. For the last layout E, the angular velocities are still small

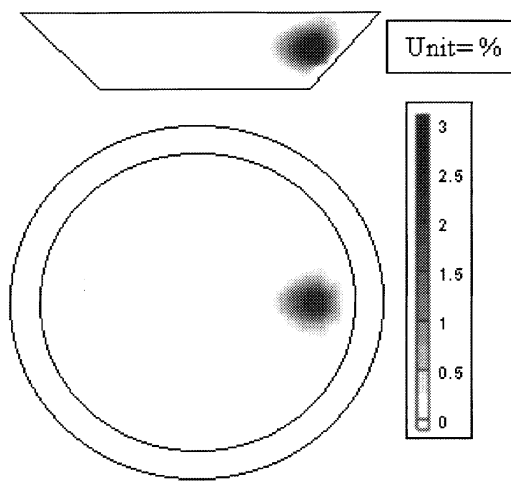

(a)

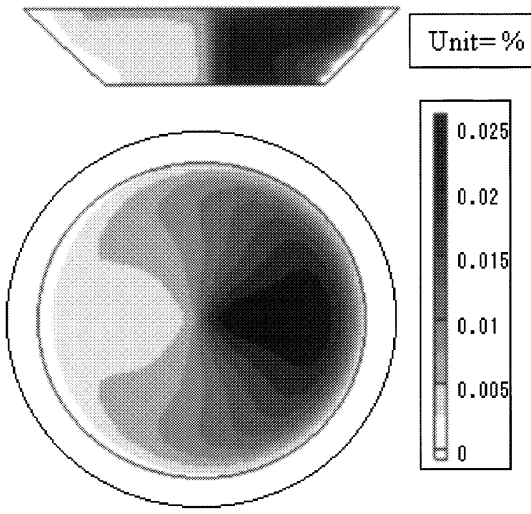

(c)

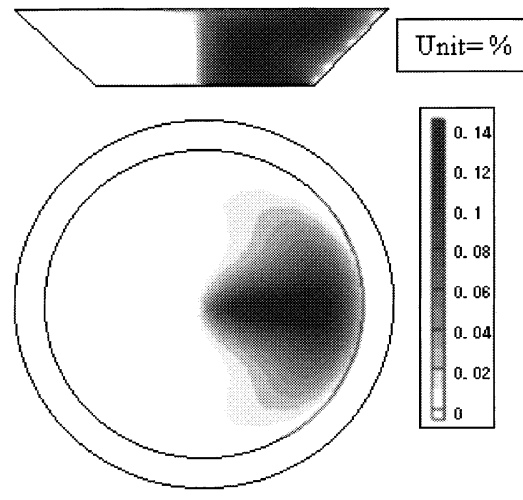

(b)

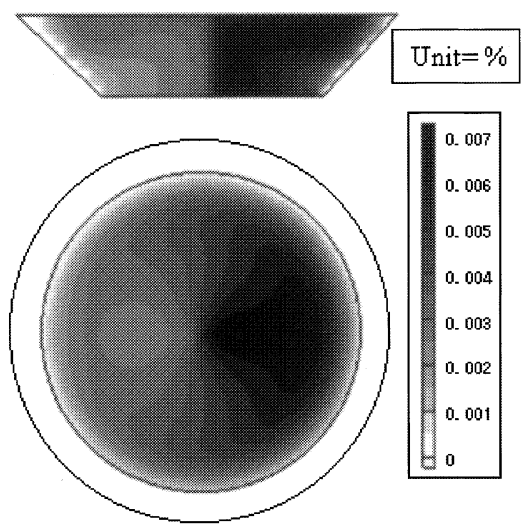

(d)

Fig. 8. Variation of tracer concentration in main vertical section and horizontal section $(Z=0.5 H)$ with the times for layout A, $Q=3 \mathrm{Nl} / \mathrm{min}$. (a) $2 \mathrm{~s}$, (b) $10 \mathrm{~s}$, (c) $30 \mathrm{~s}$, and (d) $50 \mathrm{~s}$. 


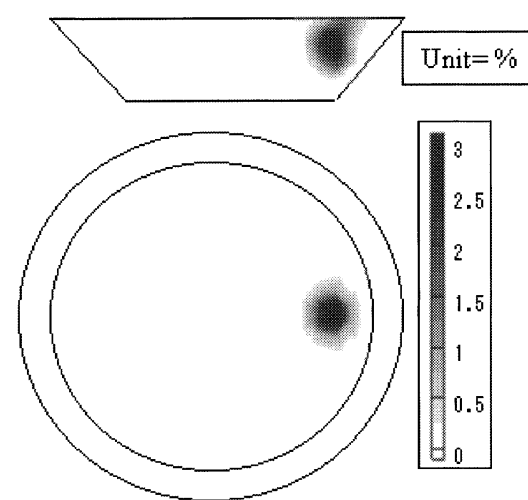

(a)

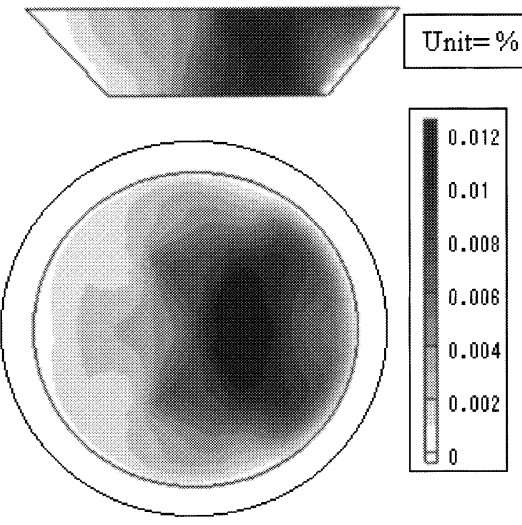

(c)

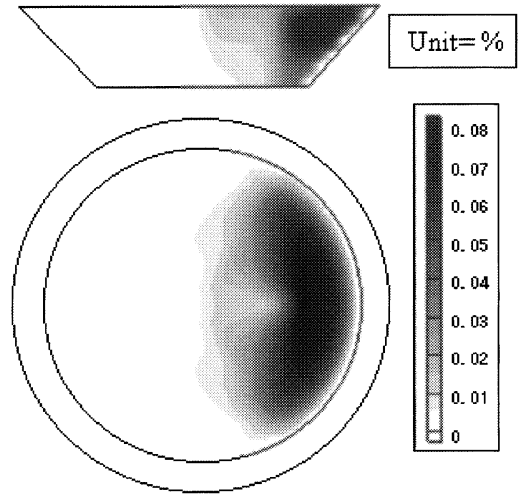

(b)

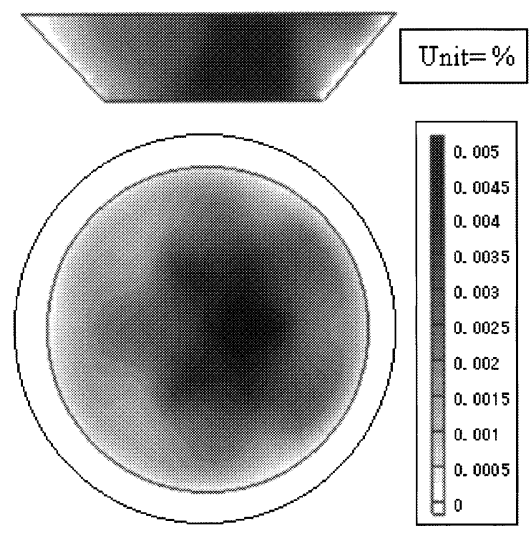

(d)

Fig. 9. Variation of tracer concentration in main vertical section and horizontal section $(Z=0.5 H)$ with the times for layout B, $Q=3 \mathrm{~N} l / \mathrm{min}$. (a) $2 \mathrm{~s}$, (b) $10 \mathrm{~s}$, (c) $30 \mathrm{~s}$, and (d) $50 \mathrm{~s}$.

Table 3. Mixing times for various layouts $(Q=3 \mathrm{~N} l / \mathrm{min})$.

\begin{tabular}{llllll}
\hline Layout & A & B & C & D & E \\
\hline Measured mixing time, s 96 & 63 & 292 & 58 & 87 \\
Computed mixing time, s & 102 & 67 & 299 & 63 & 93 \\
\hline
\end{tabular}

Table 4. Predicted mean velocities of axial, radial, and angular directions in a $1 / 7$ scale water model of a 30 -ton EAF for various layouts $(Q=3 \mathrm{~N} l / \mathrm{min}$, unit $=\mathrm{m} / \mathrm{s})$.

\begin{tabular}{llllll}
\hline & $|u|$ & $|v|$ & $|w|$ & $\left|V_{\text {av }}\right|$ & $\left|V_{\mathrm{m}}\right|$ \\
\hline Layout A & 0.0313 & 0.0173 & 0.0000 & 0.0358 & 0.3812 \\
Layout B & 0.0101 & 0.0074 & 0.0070 & 0.0143 & 0.2311 \\
Layout C & 0.0038 & 0.0040 & 0.0038 & 0.0067 & 0.1031 \\
Layout D & 0.0247 & 0.0102 & 0.0070 & 0.0276 & 0.2124 \\
Layout E & 0.0214 & 0.0110 & 0.0021 & 0.0234 & 0.2035 \\
\hline
\end{tabular}

and the mixing time is also longer than that of the D.

\section{Conclusions}

The bottom stirring EAF have been studied by experiment and mathematical model for a one seventh scale of 30-ton furnace. The conclusions are summarized as follows:

(1) There is a good agreement between computed flow velocities and experiment for layouts $\mathrm{A}$ and $\mathrm{B}$, and computed mixing times are also closed to those of measurement.

(2) Flow patterns are strongly dependent on the number and locations of plugs. As the plugs are moved off-center, the angular velocities increase, and improve the stirring ef- ficiency significantly.

(3) The variation of mixing time is not obvious with the increasing of plug diameter. The stirring efficiency is getting better if plug number is increased and plugs are distributed in deep and off-central region in vessel. The mixing times depend on the tracer injection point and plug arrangement.

(4) In this study, the layout B and D are recommended for the furnace d esign.

\section{Nomenclature}

$C:$ the concentration of tracer

$d$ : plug diameter $(\mathrm{m})$

$H$ : depth of water in vessel (m)

$g$ : acceleration due to gravity $\left(\mathrm{m} / \mathrm{s}^{2}\right)$

$k$ : turbulent kinetic energy $\left(\mathrm{m}^{2} / \mathrm{s}^{2}\right)$

$p: \quad$ pressure $\left(\mathrm{N} / \mathrm{m}^{2}\right)$

$R:$ top diameter of vessel in water level (m)

$Q: \quad$ airflow rate $(\mathrm{N} l / \mathrm{min})$

$t:$ time (s)

$r, \theta, z:$ the cylindrical polar coordinates (m)

$u, v, w$ : the velocity component in $r, \theta, z$ direction $(\mathrm{m} / \mathrm{s})$

$V_{\mathrm{av}}:=\sqrt{u^{2}+v^{2}+w^{2}}(\mathrm{~m} / \mathrm{s})$

$V_{\mathrm{m}}$ : the maximum velocity in the flow field $(\mathrm{m} / \mathrm{s})$

Greek letter

$\alpha$ : gas volume fraction

$\rho, \rho_{\mathrm{g}}, \rho_{\mathrm{L}}:$ mixture, gas, and liquid density $\left(\mathrm{kg} / \mathrm{m}^{3}\right)$ 
$\mu_{1}, \mu_{\mathrm{t}}, \mu_{\mathrm{e}}$ : laminar, turbulent, and effective viscosity ( $\mathrm{N}$. $\left.\mathrm{s} / \mathrm{m}^{2}\right)$

$\varepsilon$ : the rate of dissipation of turbulent kinetic energy $\left(\mathrm{m}^{2} / \mathrm{s}^{2}\right)$

$\sigma:$ laminar Schmidt number

$\sigma_{\mathrm{t}, \mathrm{C}}:$ turbulent Schmidt number

$\sigma_{k}, \sigma_{\varepsilon}$ : Schmit number of turbulent kinetic energy and its dissipation

\section{REFERENCES}

1) J. H. Grevet, J. Szekely and N. El-Kaddah: Int. J. Heat Mass
Transfer, 25 (1982), 487.

2) Y. Sahai and R. I. L. Guthrie: Metall. Trans. B, 13B (1982), 193.

3) Y. Sahai and R. I. L. Guthrie: Metall. Trans. B, 13B (1982), 203.

4) Baokuan Li, Jicheng He, and Zhongwu Lu: Acta Metall. Sin. (China), 30 (1993), B143.

5) A. H. Castilljeos and J. K. Brimacombe: Metall. Trans. B, 18B (1987), 659.

6) S. Joo and R. I. L.Guthrie: Metall. Trans. B, 23B (1992), 765.

7) B. E. Launder and D. B. Spalding: Comp. Math. Appl. Mech. Eng., 3 (1974), 269. 